\title{
Comprehensive study of the potential energy surface minima of a monolayer on (111) surface
}

\author{
Alexandre Tkatchenko* \\ Departamento de Química, División de Ciencias Básicas e Ingeniería, Universidad Autónoma Metropolitana-Iztapalapa, \\ Avenida San Rafael Atlixco 186 Col. Vicentina, AP. 55-534, 09340, Distrito Federal, Mexico \\ (Received 5 October 2006; revised manuscript received 23 December 2006; published 13 February 2007)
}

\begin{abstract}
The potential energy landscape of a monolayer adsorbed on well-ordered (111) surface is analyzed for periodic cells with a variable number of adsorbate $\left(N_{a d s}\right)$ and substrate $\left(N_{\text {sub }}\right)$ particles. The atom-surface potential is described by the first Fourier series term with variable corrugation, while the lateral interaction in the monolayer is modeled by a repulsive exponential term. Special attention is devoted to the determination of the total number of minima for given $N_{a d s}$ and $N_{s u b}$ and the probability of relaxation to the global minimum in each of the unit cells, as well as the construction of the lowest energy versus coverage curve as a function of the atom-surface potential corrugation. We find that the global appearance of the energy landscape in the majority of the unit cells is particularly simple, characterized by the global minimum positioned in a very wide basin and the high-energy minima forming a tail structure. However, this rule is broken for several unit cells when the corrugation of the atom-surface potential becomes large, making the location of the global minimum a rather difficult task. Despite the simplicity of our model, phase transitions from commensurate to striped incommensurate to hexagonal incommensurate rotated structures are observed.
\end{abstract}

DOI: 10.1103/PhysRevB.75.085420

PACS number(s): 68.35.Md, 71.15.Ap, 31.15.Ew

\section{INTRODUCTION}

The potential energy landscapes of different systems, such as clusters, biomolecules, and glasses, have attracted much attention over the last few years. ${ }^{1-6}$ The availability of high quality interaction potentials makes possible the detailed analysis of minima and saddle points in order to gain valuable insight into the kinetics and dynamics of these systems. On the other hand, adsorbates on well-ordered surfaces represent a system where the interaction potentials are available in very few cases, mainly for rare gases adsorbed on metal surfaces. $^{7-9}$ For many chemisorption systems, not even a phenomenological picture of interactions is available yet.

Due to this lack of knowledge regarding the forces present in adsorbate-substrate systems, many different "toy" models have been proposed and extensively studied so far. One of the most well-known and studied ones is the FrenkelKontorova model, ${ }^{10}$ where the interaction between adjacent atoms is modeled by springs. Once the correct force constant for a given system is known, the Frenkel-Kontorova model can be successfully used for simulation of the ground state of the monolayer-surface system. This model has been employed for simulation of reconstruction of the $\mathrm{Ni}(100)$ surface, ${ }^{11} \mathrm{Cu}$ atoms adsorbed on the $\mathrm{Ru}(1000)$ surface, ${ }^{12}$ and herringbone reconstruction of $\mathrm{Au}(111),{ }^{13}$ just to mention a few previous works. Another kind of studies includes those which use the Lennard-Jones potential for the description of monolayer-surface interactions and which have been frequently employed in the study of the ground state of monolayers on the (100) surface. ${ }^{14-16}$

Monolayers formed at well-ordered surfaces show a wide variety of ordered structures: simple commensurate, uniaxially compressed, and complex incommensurate with domain walls among others. ${ }^{7,17,18}$ Even when analytic expressions for the interactions of a monolayer-surface system are known, i.e., for $\mathrm{Xe}-\mathrm{Pt}(111),{ }^{19,20}$ the determination of the global minimum is still a formidable task. ${ }^{21}$ That is, although molecular dynamics could be employed with a large system size to determine the most probable minimum of an adsorbatesubstrate system, ${ }^{22}$ this approach suffers from the finite-size effect and requires an initial guess, making the search process biased. Furthermore, the adsorbate-substrate system is usually described by a fixed finite unit cell under periodic boundary conditions, where the ground state and phase transitions are obtained with the change in model parameters. However, this approach is rather inadequate whenever precise characterization of stable structures is desired. For instance, it would require a very large unit-cell size to distinguish between high-order commensurate and incommensurate structures, thus making a detailed study of the potential energy surface in this unit cell computationally prohibitive. An unbiased search method for the global minimum and commensurate-incommensurate phase transitions of an adsorbate-substrate system should be able to obtain the global minimum in a series of unit cells with variable coverage.

In comparison with true three-dimensional (3D) systems, such as clusters and biomolecules, the investigation of the potential energy surfaces (PES) of monolayer-surface systems possesses several advantages. Specific cells with different $N_{a d s}$ and $N_{\text {sub }}$ and defined coverage $\theta \in 1, \frac{1}{3}, \frac{1}{4}, \ldots$ can be generated, where the global minimum is known a priori and consists of every particle positioned on the equivalent most stable site. This fact can be used to obtain necessary heuristics about the PES of cells with given $N_{a d s}$. In the case of 3D systems, one can never be sure when the global minimum has been reached, thus requiring complex heuristics and detailed knowledge of the PES to propose and "prove" that a certain minimum is the global one. Another advantage is that the atom-surface potential of the monolayer-surface system can be systematically varied and trends can be obtained for different terms of the corresponding Fourier expansion. Furthermore, the restricted two-dimensional symmetry of the monolayer should reduce the number of minima from what is expected in 3D for an equivalent number of particles. 
In the present work, the potential energy landscape of a model monolayer-surface system is analyzed in detail. The atom-surface potential is described by the first Fourier series term with variable corrugation, while the lateral interaction in the monolayer is modeled by a repulsive exponential term. The focus is on the determination of the total number of minima and the probability of relaxation to the global minimum for a series of unit cells of the (111) surface. It is shown that even when the number of minima of a given unit cell is very large, the global minimum can be found with an unbiased search starting from random configurations of monolayer particles. Furthermore, we obtain the global minimum of an extensive sample of unit cells for the coverage interval $0.33 \leqslant \theta \leqslant 0.5$. This allows us to propose the most probable minimum structures of our model system and a possible sequence of phase transitions between them.

\section{THE MODEL AND SYSTEMS}

It is well known that when the corrugation of the surface potential is negligible, the ground state of the monolayer is trivial, adopting a perfect hexagonal arrangement with a spacing given by the minimum of the lateral interaction potential. When either the surface corrugation or the (repulsive) lateral interaction is strong, hexagonal (in)commensurate monolayer-surface structures emerge. ${ }^{23,24}$ Therefore, it is reasonable to assume that the lowest-energy structures for a given coverage are located in cells where the formation of perfect hexagonal monolayers is possible.

A primitive cell of the (111) surface with atomic radius $R=1$ is spanned by two vectors, $v_{1}=(2,0)$ and $v_{2}=(1, \sqrt{3})$, with $60^{\circ}$ angle between them. Any expanded nonprimitive cell is then obtained as a linear combination of these two vectors: $V_{1}=(2 a+b,-\sqrt{3} b)$ and $V_{2}=(a+2 b, \sqrt{3} a)$, where $a$ and $b$ are integers. The number of atoms in such expanded cell is $N=a^{2}+b^{2}+a b$. This can be shown by packing $N$ circles of radius $R=1$ into a rhombus enclosing the unit-cell area. We restrict our study to cells with $N_{s u b}=a^{2}+b^{2}+a b$ and $N_{a d s}=A^{2}+B^{2}+A B$, where $a, b$ and $A, B$ are integers. The coverage $\left(\theta=\frac{N_{\text {ads }}}{N_{\text {sub }}}\right)$ is restricted to $0.33 \leqslant \theta \leqslant 0.5$. This covers the adsorption coverage region of many real systems, such as rare gases and halogens on metal surfaces. ${ }^{25,26}$

We generate cells with all possible combinations of $N_{a d s}$ and $N_{s u b}$ for $N_{a d s} \leqslant 43$ and $0.33 \leqslant \theta \leqslant 0.5$. These parameters give rise to 128 different cells. In what follows, these cells will be called $C \frac{N_{a d s}}{N_{s u b}}$. Standard periodic boundary conditions are used in the $x$ and $y$ directions for all unit cells.

The atom-surface potential is represented by the first term of the Fourier series, $, 9,27$

$$
\begin{aligned}
V(x, y)= & 2 V_{G_{0}}\left\{\cos \left(G_{0} y\right)+\cos \left[G_{0}\left(\frac{\sqrt{3}}{2} x-\frac{1}{2} y\right)\right]\right. \\
& \left.+\cos \left[G_{0}\left(-\frac{\sqrt{3}}{2} x-\frac{1}{2} y\right)\right]\right\},
\end{aligned}
$$

where $G_{0}=\frac{4 \pi}{\sqrt{3} l}, l=2$ is the surface lattice constant, and $V_{G_{0}}$ is the first Fourier coefficient. The variation is allowed from $V_{G_{0}}=-1.111$ to $V_{G_{0}}=-3.888$, corresponding to the potential corrugation from 10 to 35 arbitrary energy units. The lateral interaction between the monolayer atoms is modeled by a simple exponential term $C \exp (-D x)$ (Ref. 28). Although, it is a simplification when compared to real systems, such model has been used successfully for the description of iodine adsorbed on the Pt(111) surface. ${ }^{28}$ Furthermore, the use of such simple expression allows us to decompose the total interaction potential into attractive (adsorbate-substrate) and repulsive (adsorbate-adsorbate) parts. This model should be able to represent real systems for a small variation in coverage, as the $C$ and $D$ parameters can be tuned to represent any force constant for a given interatomic distance. The parameters for the lateral interaction are taken to be $C=8 \times 10^{5}$ and $D=4$. These particular values have been chosen to simulate strong repulsion in the monolayer. The cutoff for calculation of the lateral interaction is set to 20 (in surface lattice constant units). For each cell, this has been accomplished by changing the number of adjacent cells taken into account for the calculation of the lateral interaction.

The main goals of our study are as follows.

(1) Analysis of the potential energy landscape in cells with $\theta=\frac{1}{3}$. The lowest-energy structure in these cells regardless of $N_{a d s}$ and $N_{\text {sub }}$ is the $(\sqrt{3} \times \sqrt{3}) R 30^{\circ}$ one with one atom per primitive unit cell. This analysis will allow a precise determination of the probability of relaxation to the global minimum as a function of $N_{a d s}$.

(2) Enumeration of the total number of minima and deduction of the probability of relaxation to the global minimum as a function of the atom-surface potential corrugation $\left(V_{G_{0}}\right)$ and coverage (change of $N_{\text {sub }}$ for a fixed $N_{a d s}$ ).

(3) Determination of the lowest energy vs coverage curve as a function of the atom-surface potential corrugation and identification of phase transitions (i.e., commensurate to incommensurate).

To fulfill these objectives, we perform an excessively large number of conjugate-gradients local optimizations in each $C \frac{N_{a d s}}{N_{s u b}}$ cell as needed. The positions of $N_{a d s}$ adsorbate particles are initialized randomly before each conjugategradient optimization. For each part of our study, a specific strategy is used to ensure that a sufficient number of optimizations have been performed before drawing conclusions. The algorithm for choosing this specific strategy will be explicitly stated in the next section.

Although several robust algorithms are available for global optimization such as basin hopping, ${ }^{29}$ genetic algorithms, ${ }^{30,31}$ simulated annealing, ${ }^{32}$ and others, the main interest of this work is the characterization of the potential energy surface minima of an adsorbate-substrate system. Due to the fact that little is known about the topography and topology of these systems, such "brute force" approach seems to be justified. Moreover, it will be shown that although the total number of minima for a given $C \frac{N_{a d s}}{N_{s u b}}$ cell can be large, the probability of finding the global minimum is frequently quite large as well. This fact provides further support to the brute force approach adopted in this work.

\section{RESULTS}

Due to the effect of the atom-surface holding potential, the global minimum of a cell with $\theta=\frac{1}{3}$ is the $(\sqrt{3}$ 


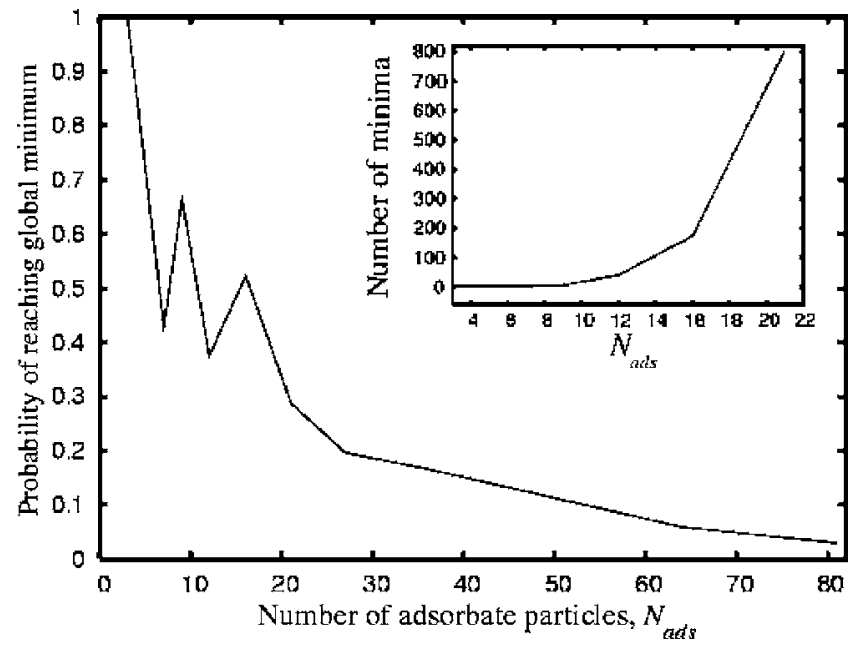

FIG. 1. Plot of the probability of reaching the global minimum vs the number of adsorbate particles $N_{a d s}$ for cells with $\theta=\frac{1}{3}$ and $V_{G_{0}}=-1.111$. The inset shows the total number of minima found for given $N_{a d s}$ in the same unit cells.

$\times \sqrt{3}) R 30^{\circ}$ structure with one atom basis, regardless of $N_{a d s}$ and $N_{s u b}$. This comes in handy when identifying how probable the process of finding the global minimum of a given $C \frac{N_{a d s}}{N_{\text {sub }}}$ cell starting from a random configuration of adsorbate atoms is.

We have chosen ten different unit cells, from $C \frac{3}{9}$ to $C \frac{81}{243}$, in order to determine this probability. Figure 1 shows the probability of finding the global $(\sqrt{3} \times \sqrt{3}) R 30^{\circ}$ minimum as a function of the number of adsorbate atoms $N_{a d s}$ for $V_{G_{0}}=-1.111$. It changes from $100 \%$ for the $C \frac{3}{9}$ cell to $3 \%$ for the $C \frac{81}{243}$ cell. Note that this curve does not show simple exponential decay; instead there are two local maxima for $N_{a d s}=9$ and $N_{a d s}=16$. However, for $N_{a d s}>16$ the decay becomes exponential. Even when $N_{a d s}=81$, around 30 optimizations are enough to find the global minimum with a brute force approach. In order to better understand the magnitude of the probability of reaching the global minimum, we have also performed an extensive number of optimizations from $C \frac{3}{9}$ to $C \frac{21}{63}$ to determine the total number of minima in these cells. The algorithm we used is as follows: (1) Perform 10000 optimizations and identify different minima. ${ }^{33}$ (2) Perform additional 10000 optimizations and, if no other local minimum has been found, stop; otherwise go to step (1). It is worth mentioning that the above algorithm required around $5 \times 10^{5}$ steps to find all minima in the $C \frac{21}{63}$ cell due to the well-known exponential growth in the number of minima with the increase in the number of simulated particles. ${ }^{29,34,35}$ This is illustrated in the inset of Fig. 1. We have identified 803 distinct minima in the $C \frac{21}{63}$ cell; however, the probability of finding the global minimum for this cell starting from a random configuration is around $29 \%$. Therefore, it seems that the global minimum is well defined and located in a very broad basin of the potential energy surface. The densities of minima curves are shown in Fig. 2 for $C \frac{9}{27}, C \frac{12}{36}, C \frac{16}{48}$, and $C \frac{21}{63}$. Once again, one can clearly distinguish the global $(\sqrt{3}$ $\times \sqrt{3}) R 30^{\circ}$ minimum as the most likely one. The second closest minimum is generally far away in energy and reach-

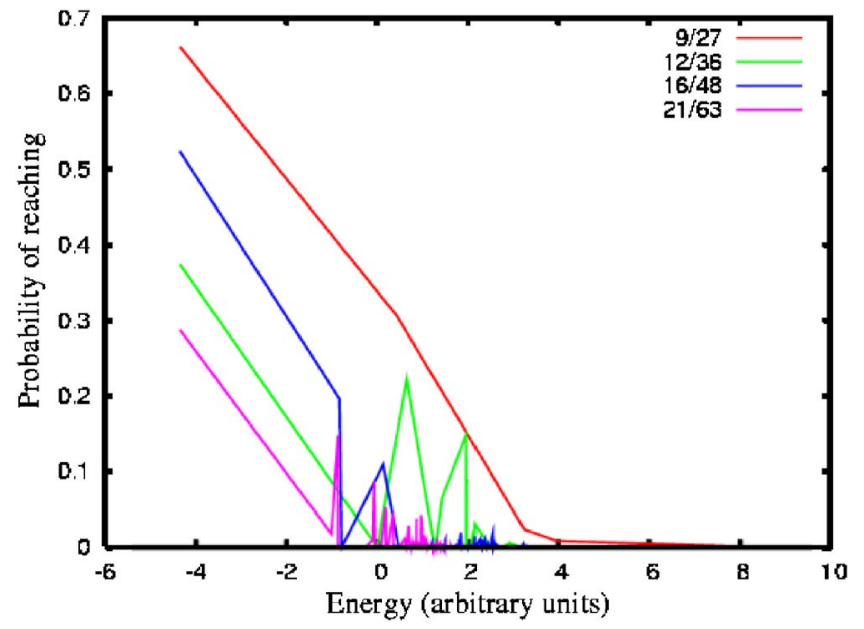

FIG. 2. (Color online) Density of minima plot for $C \frac{9}{27}, C \frac{12}{36}$, $C \frac{16}{48}$, and $C \frac{21}{63}$ cells with $V_{G_{0}}=-1.111$.

ing probability. The majority of high-energy minima form characteristic tail structure and lie far away in energy from the global minimum.

Another interesting question is how the total number of minima and the probability of reaching the global minimum are affected by the corrugation of the atom-surface potential. To answer this question, we changed $V_{G_{0}}$ from -1.111 to -3.888 and repeated the simulations in cells with $\theta=\frac{1}{3}$. The total number of minima increased dramatically when going from $V_{G_{0}}=-1.111$ to $V_{G_{0}}=-3.888$. For $C \frac{12}{36}$, it increased from 40 to 1044 . However, the probability of reaching the global minimum is only slightly affected: it decreases from $37 \%$ to $31 \%$. In the case of a larger $C \frac{21}{63}$ cell, the global minimum is found in $29 \%$ of the runs for $V_{G_{0}}=-1.111,24 \%$ for $V_{G_{0}}=$ -1.666 , and $14 \%$ for $V_{G_{0}}=-3.888$. These results indicate that even while the total number of minima may be very large for a given unit cell, the global minimum can be easily found by simple unbiased search starting from random configurations.

In order to investigate the effect of the repulsion gradient on the potential energy landscape of our model system, we have studied the total number of minima when $N_{a d s}$ is kept constant and $N_{s u b}$ is changed. An illustrative example is the case of the $C \frac{21}{49}$ cell in comparison to the previously studied $C \frac{21}{63}$ one. The total number of minima in the latter is 803 for $V_{G_{0}}=-1.111$, while for the former it is only 33 . The reduction in the number of minima is dramatic due to the increased repulsion between the atoms in the monolayer. When the repulsion is increased, the monolayer atoms become more restricted in their movement, explaining the reduced number of minima. On the contrary, the increase of $V_{G_{0}}$ allows the adsorbate atoms to relax into more favorable sites, thus increasing the possibility of formation of new minimum structures. However, the effect of both changes on the probability of reaching the global minimum seems to be small. This fact will be used below for constructing the lowest energy versus coverage curve and for investigating the structures and phase transitions of our model system.

So far, we have analyzed the minima in cells with $\theta=\frac{1}{3}$ and identified simple rules to determine the rate of relaxation 


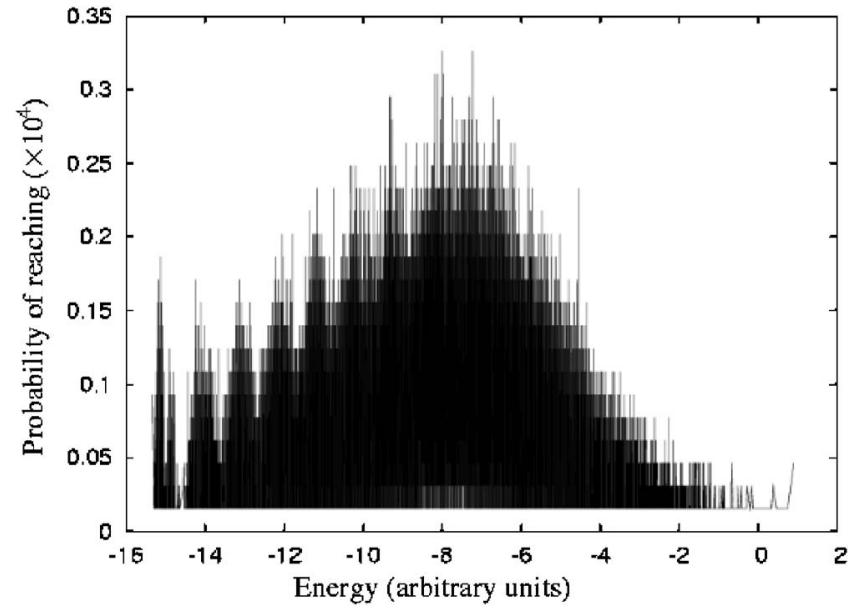

FIG. 3. Density of minima plot for $C \frac{37}{112}\left(V_{G_{0}}=-3.888\right)$ with global minimum being not the most probable.

to the global minimum. However, it is not clear how general these rules are and whether they can be generally applied to any $C \frac{N_{a d s}}{N_{\text {sub }}}$ cell. To answer this question, we have performed simulations in 128 different cells, with $3 \leqslant N_{a d s} \leqslant 43$ and $0.33 \leqslant \theta \leqslant 0.5$. For each unit cell, the following procedure was employed: (1) 1000 optimization runs have been accomplished and the minima have been sorted from the lowest to the highest ones. (2) Step (1) is repeated until the lowestlying minimum has been found at least five times. Although this procedure is not guaranteed to find the global minimum, it has done so for all cells with $\theta=\frac{1}{3}$. We have also randomly selected ten cells with $\theta \neq \frac{1}{3}$ and doubled the number of runs from the procedure described above. In all cases, the lowestlying minimum did not change. Therefore, I believe that the probability of finding the global minimum with this procedure is quite high. For $V_{G_{0}}=-1.111$ and $V_{G_{0}}=-1.666$, the global minimum has been found in almost all cells within 1000 runs; in some of them, it required 2000 runs to obtain the lowest-lying minimum five times. However, this situation changed for $V_{G_{0}}=-3.888$. The global minimum in the vast majority of cells has been found within 1000 optimizations; however several (6) cells showed difficulty in finding the global minimum and required more than 10000 iterations. For at least three cell $\left(C \frac{36}{109}, C \frac{39}{109}\right.$, and $\left.C \frac{37}{112}\right)$, we have not been able to find the lowest-lying minimum five times even after 50000 runs. In Fig. 3, the minima density plot for $C \frac{37}{112}$ is depicted for $V_{G_{0}}=-3.888$. The structures with the highest reaching probability are those in the middle of the plot; however, no particular structure is favored in striking difference with cells of $\theta=\frac{1}{3}$.

Another interesting comparison can be made for the $C \frac{25}{75}$ and $C \frac{25}{73}$ cells, whose density of minima is shown in Fig. 4. The coverage of both cells is almost identical, differing only in two substrate particles. However, it can be seen that while the $C \frac{25}{75}$ possesses a familiar structure of minima (welldefined global minimum and high-energy minima forming a tail structure), the $C \frac{25}{73}$ cell possesses many candidates with similar reaching probability (and very small compared to the $C \frac{25}{75}$ case). It would be interesting to find the reason for such

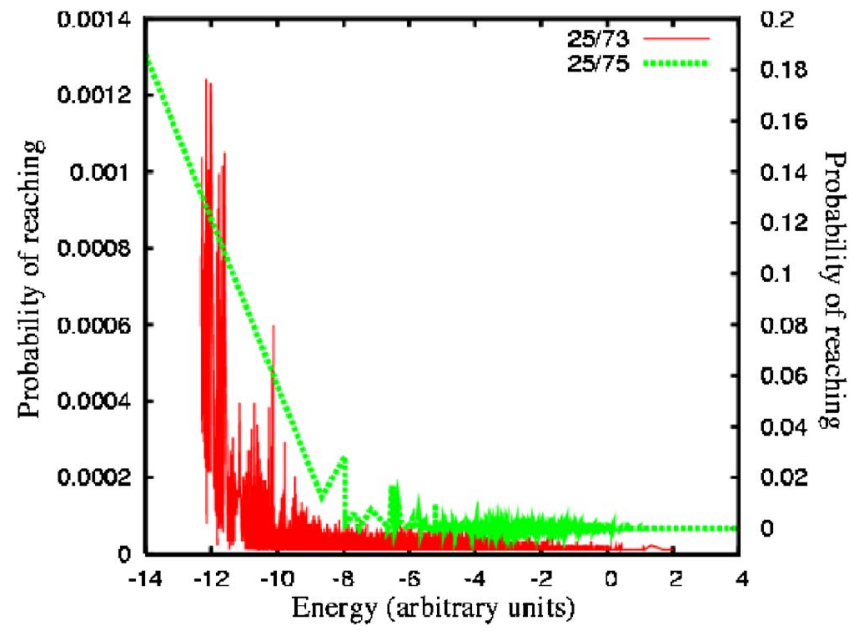

FIG. 4. (Color online) Density of minima plot for $C \frac{25}{73}$ and $C \frac{25}{75}$ $\left(V_{G_{0}}=-3.888\right)$. Scales on the left-hand and right-hand sides correspond to the $C \frac{25}{73}$ and $C \frac{25}{75}$ cells, respectively. Notice the extremely reduced scale for the $C \frac{25}{73}$ cell.

drastic change for apparently similar cells. One should note that even if no warranty exists that the global minimum has been found in the $C \frac{25}{73}$ cell, the relatively high density of minima indicates that the lowest-lying minimum should be close enough to the global one after a sufficiently large number of runs.

Finally, we proceed to calculate the global minimum energy versus coverage curve for different corrugations of the atom-surface potential, encouraged by the fact that the global minimum can be easily obtained in most cells for $3 \leqslant N_{a d s}$ $\leqslant 43$ and $0.33 \leqslant \theta \leqslant 0.5$. We follow the same algorithm as employed in the previous paragraph. The minimum energy curve is depicted in Fig. 5 using $V_{G_{0}}=-1.111$ for $0.33 \leqslant \theta$ $\leqslant 0.44$. As expected, on a large scale the energy is getting higher with an increase in coverage. However, many local minima can be distinguished for the simulated coverage range. The first minimum on the left side (global minimum) is the simple commensurate $(\sqrt{3} \times \sqrt{3}) R 30^{\circ}$ structure. The ball models for some intermediate minima are shown in Fig.

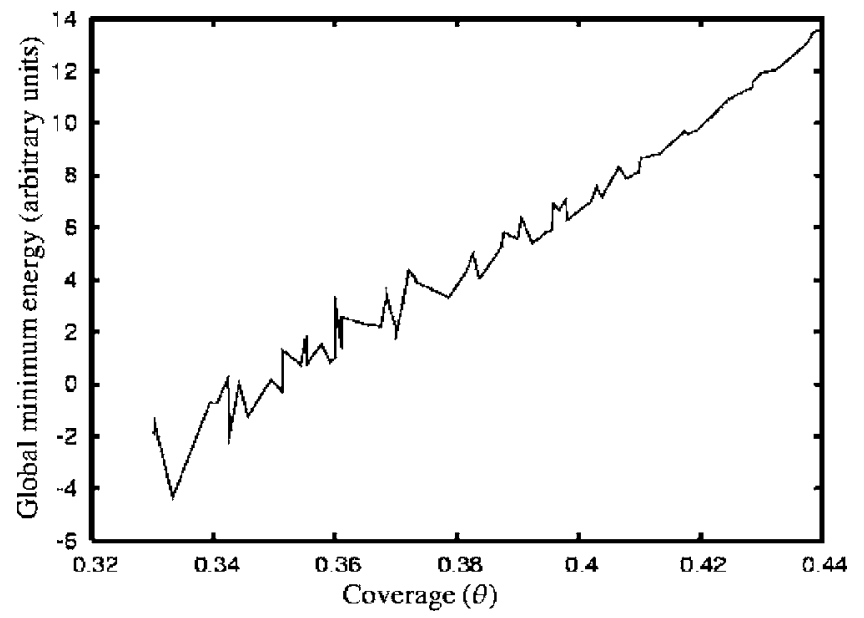

FIG. 5. Global minimum energy versus coverage plot for cells with $3 \leqslant N_{a d s} \leqslant 43$ and $0.33 \leqslant \theta \leqslant 0.5$ with $V_{G_{0}}=-1.111$. 

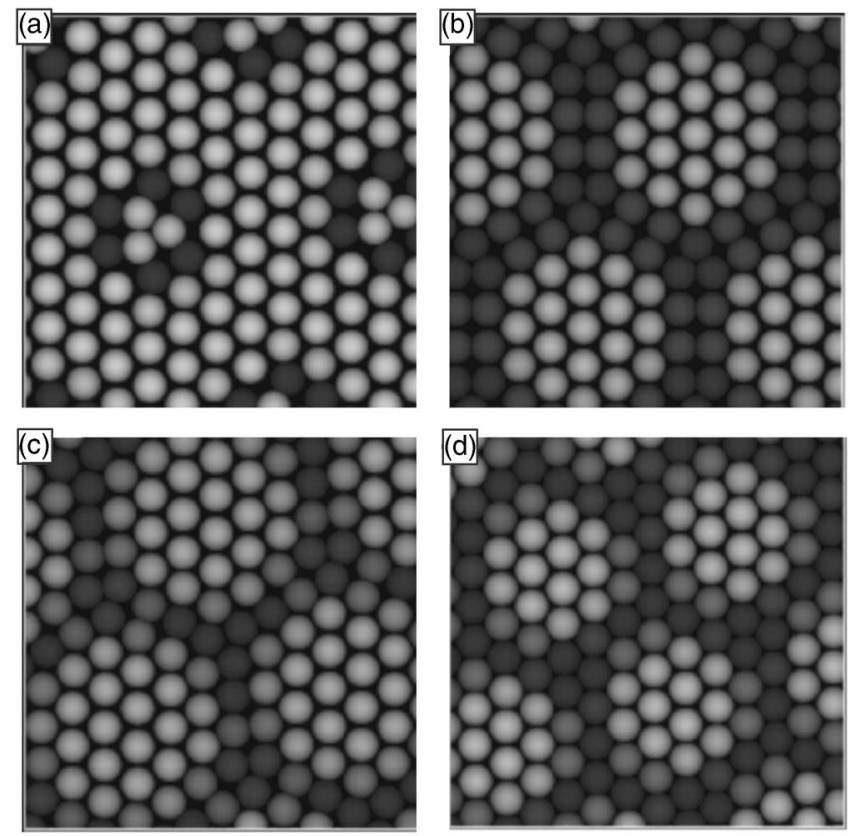

FIG. 6. Topographic view of different global minimum structures (GMSs) with $V_{G_{0}}=-1.111$ : (a) GMSs of the $C \frac{39}{111}$ cell, (b) GMSs of the $C \frac{37}{100}$, (c) GMSs of the $C \frac{39}{103}$, and (d) GMSs of the $C \frac{28}{67}$. The stable structures range from commensurate $(\sqrt{3} \times \sqrt{3}) R 30^{\circ}$ (not shown here), to striped incommensurate (SI) in the $C \frac{39}{111}$ and $C \frac{37}{100}$ cells, between SI and hexagonal incommensurate rotated (HIR) for the $C \frac{39}{103}$ cell, and HIR for the $C \frac{28}{67}$ cell. The images were generated using ALSA software (Ref. 36).

6. One can clearly see that increasing the coverage from $\theta$ $=\frac{1}{3}$ makes the striped incommensurate $(\mathrm{SI})$ structures stable. The $(\sqrt{3} \times \sqrt{3}) R 30^{\circ}$ domains are separated by domain walls. The structure of the domain walls depends on the coverage, changing from triangular shape for $C \frac{37}{108}$, hexagonal shape for $C \frac{39}{111}$, to heavy domain walls for $C \frac{37}{103}$ and $C \frac{37}{100}$. For $\theta$ $\geqslant 0.371$, a phase transition to hexagonal incommensurate rotated (HIR) structures occurs. The reason for this transition is that the density of domain walls exceeds certain critical value and, even if the monolayer-surface energy is increased for rotated incommensurate structures, the repulsive lateral interaction makes the monolayer adopt a hexagonal arrangement. After $\theta=0.42$, the curve in Fig. 5 maintains a linear shape.

It is not clear what the effect of the $N_{a d s} \leqslant 43$ restriction on the minimum energy curve is. Therefore, we have performed additional simulations for $43 \leqslant N_{a d s} \leqslant 81$ and obtained the global minimum energy for all unit cells, taking advantage of the fact that relaxation to the global minimum for $V_{G_{0}}=-1.111$ requires a small number of conjugategradient optimizations even for $N_{a d s}=81$. The curve becomes more complex than the one in Fig. 5, with a lot more intermediate minima. However, the overall shape and phase transitions seem to coincide with the simulations for the reduced set of cells, and the conclusions of our study are not modified by this fact. Moreover, these differences between smaller and larger sets of cells pose an interesting question: "what is the limiting value of $N_{a d s}$ in order to reach an accurate descrip-

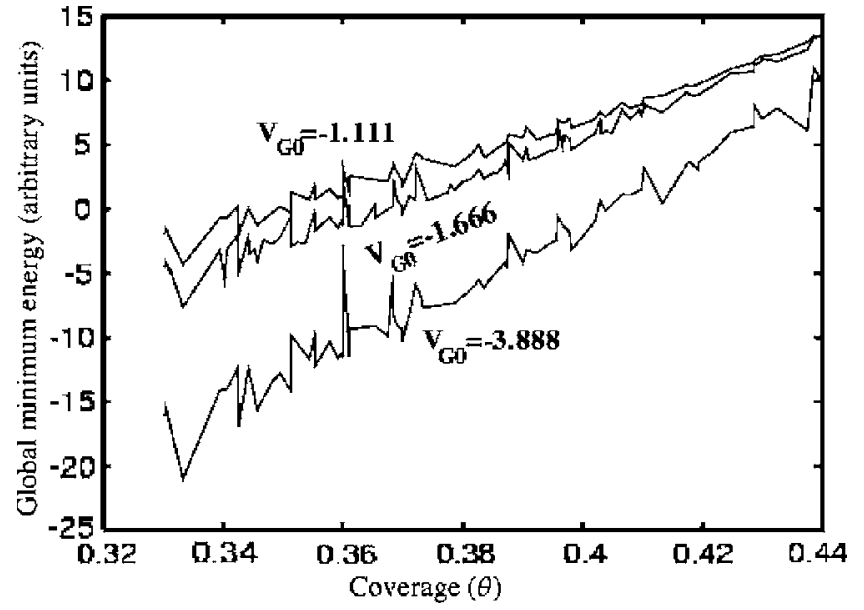

FIG. 7. Global minimum energy versus coverage plot for cells with $3 \leqslant N_{a d s} \leqslant 43,0.33 \leqslant \theta \leqslant 0.5$, and $V_{G_{0}}=-1.111, V_{G_{0}}=-1.666$, and $V_{G_{0}}=-3.888$. The sudden jumps at some points of the curve are due to cells with the same coverage (e.g., $C \frac{3}{7}$ and $C \frac{21}{49}$ ) but with different energies.

tion of incommensurate phases?" The answer is clearly dependent on the particular interaction model, but it is worth pursuing additional work to analyze this question in detail.

The global minimum energy vs coverage curves are plotted in Fig. 7 for $V_{G_{0}}=-1.111, V_{G_{0}}=-1.666$, and $V_{G_{0}}$ $=-3.888$. The first observation is that the energy of the monolayer-surface system goes down while the atom-surface potential corrugation is increased, as expected. The simple commensurate $(\sqrt{3} \times \sqrt{3}) R 30^{\circ}$ arrangement also becomes more stable in comparison to the surrounding structures when the corrugation is higher. The curves for $V_{G_{0}}=-1.111$ and $V_{G_{0}}=-1.666$ are similar, with just a few differences in local minima. The curve for $V_{G_{0}}=-3.888$ is more complex than for other values of $V_{G_{0}}$ and local minima can be observed even for $\theta \geqslant 0.44$ (not shown in Fig. 7). While the $(\sqrt{3} \times \sqrt{3}) R 30^{\circ}$ is the only commensurate structure observed for $V_{G_{0}}=-1.111$ and $V_{G_{0}}=-1.666$, another commensurate $(4 \times 4)$ structure is found for $V_{G_{0}}=-3.888$ at $\theta=0.438$, depicted in Fig. 8 (it exists at least for $N_{\text {ads }} \leqslant 43$ ). However, this structure deviates from the simplest hexagonal arrangement, possessing square and hexagonal "islands." Despite these
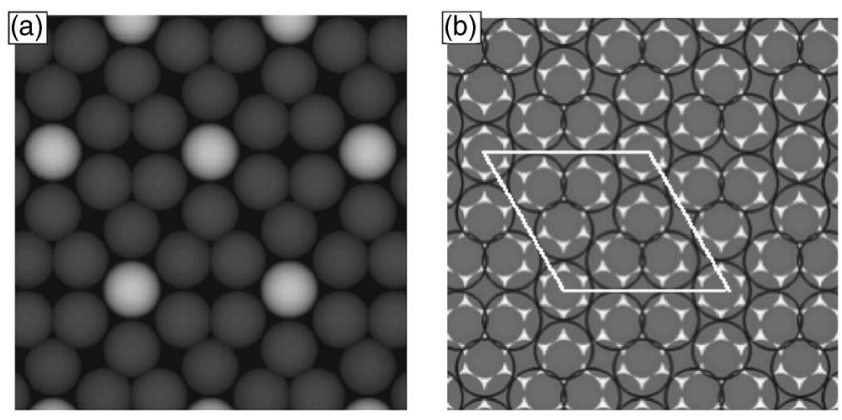

FIG. 8. The model for the $(4 \times 4)$ commensurate structure $(\theta$ $=0.438$ ) obtained with $V_{G_{0}}=-3.888$. (a) Topographic image. (b) Transparent circle model with the unit cell marked by a white parallelogram. 
differences between stable arrangements for different corrugations of the atom-surface potential, the same kind of phase transitions could be observed for any value of $V_{G_{0}}$ : from commensurate $(\sqrt{3} \times \sqrt{3}) R 30^{\circ}$ to SI to HIR.

For the sake of completeness, we have also studied the effect of the higher-order atom-surface Fourier terms on the potential energy minima. We set $V_{\sqrt{3} G_{0}}=-0.1 V_{G_{0}}$ with $V_{G_{0}}=-0.111$ and obtain the global minimum energy versus coverage curve similar to the one depicted in Fig. 5. The appearance of the curve is similar; however, the commensurate $(\sqrt{7} \times \sqrt{7}) R 19.1^{\circ}$ and $(\sqrt{19} \times \sqrt{19}) R 23.4^{\circ}$ structures become the most stable for $\theta=\frac{3}{7}$ and $\theta=\frac{7}{19}$, respectively, instead of higher-order commensurate structures, which are the most stable for a one-term Fourier model. For a detailed analysis of this particular case, see Ref. 24.

\section{CONCLUDING DISCUSSION}

A comprehensive (and somewhat exhaustive) analysis of the potential energy surface minima has been performed for a model monolayer-surface system. A large set of cells has been generated, where enumeration of local minima and the rate of relaxation to the global minimum has been obtained for a variety of interaction potential models. The energy landscape in those cells seems to have a particularly simple structure with the global minimum located in a wide basin and the majority of high-lying minima forming a characteristic tail structure. Despite the simplicity of the interactions used in this work, particularly the exponential repulsion for the interaction between the monolayer atoms, a sequence of $\mathrm{C} \rightarrow \mathrm{SI} \rightarrow \mathrm{HIR}$ structures has been observed, resembling rare- gas atoms adsorbed on metal surfaces. ${ }^{37,38}$ This fact is surprising and deserves a more thorough investigation. One possible explanation is that the qualitative nature of monolayersurface structures is largely determined by the atom-surface attraction and the repulsive part of the lateral interaction potential, as has been proposed for bulk systems. ${ }^{39}$

Several interesting questions have arisen as a result of the present study.

(1) The striking difference in the potential energy landscape of similar unit cells, i.e., $C \frac{25}{73}$ versus $C \frac{25}{75}$. The reason for this difference would be definitely worth additional investigation.

(2) The definition of "incommensurability" in a finitesize model. That is, significant differences in the global minimum energy vs coverage plot for smaller and larger sets of unit cells make one wonder what maximum $N_{a d s}$, after which the energy of incommensurate structures converges, is sufficient.

(3) The influence of the high-order Fourier terms on the stable structures and phase transitions is not yet clear enough.

\section{ACKNOWLEDGMENTS}

I am indebted to Pedro D. Cruz-Santiago for helping with computer scripting during this work, Marcelo Galván for stimulating discussions, and Carmen Heras for the access to MEZQUITE compute cluster in UNISON. Financial support by CONACYT and LUFAC Computación is greatly acknowledged.
*Electronic address: sanix@ixil.izt.uam.mx

${ }^{1}$ R. S. Berry, Chem. Rev. (Washington, D.C.) 93, 2379 (1993).

${ }^{2}$ J. P. K. Doye and D. J. Wales, J. Chem. Phys. 105, 8428 (1996).

${ }^{3}$ O. M. Becker and M. Karplus, J. Chem. Phys. 106, 1495 (1997).

${ }^{4}$ D. J. Wales, Science 293, 2067 (2001).

${ }^{5}$ D. J. Wales, Energy Landscapes: Applications to Clusters, Biomolecules and Glasses (Cambridge University Press, Cambridge, 2003).

${ }^{6}$ D. J. Wales and T. V. Bogdan, J. Phys. Chem. B 110, 20765 (2006).

${ }^{7}$ G. A. Somorjai, Introduction to Surface Chemistry and Catalysis (Wiley, New York, 1994).

${ }^{8}$ R. I. Masel, Principles of Adsorption and Reaction on Solid Surfaces (Wiley, New York, 1996).

${ }^{9}$ L. W. Bruch, M. W. Cole, and E. Zaremba, Physical Adsorption: Forces and Phenomena (Oxford University Press, New York, 1997).

${ }^{10}$ T. Kontorova and Y. I. Frenkel, Zh. Eksp. Teor. Fiz. 8, 1340 (1938).

${ }^{11}$ J. C. Hamilton, Phys. Rev. Lett. 88, 126101 (2002).

${ }^{12}$ J. C. Hamilton and S. M. Foiles, Phys. Rev. Lett. 75, 882 (1995).

${ }^{13}$ S. Narasimhan and D. Vanderbilt, Phys. Rev. Lett. 69, 1564 (1992)

${ }^{14}$ O. Biham, L.-W. Chen, and G. Vidali, Surf. Sci. 287, 815 (1993).
${ }^{15}$ A. Patrykiejew, S. Sokolowski, and K. Binder, Surf. Sci. Rep. 37, 207 (2000).

${ }^{16}$ A. Patrykiejew, S. Sokolowski, and K. Binder, J. Chem. Phys. 115, 983 (2001).

${ }^{17}$ A. C. Hillier and M. D. Ward, Phys. Rev. B 54, 14037 (1996).

${ }^{18}$ D. E. Hooks, T. Fritz, and M. D. Ward, Adv. Mater. (Weinheim, Ger.) 13, 227 (2001).

${ }^{19}$ J. A. Barker and C. T. Rettner, J. Chem. Phys. 97, 5844 (1992).

${ }^{20}$ L. W. Bruch, A. P. Graham, and J. P. Toennies, J. Chem. Phys. 112, 3314 (1999).

${ }^{21}$ R. D. Boutchko and L. W. Bruch, Phys. Rev. B 70, 195422 (2004).

${ }^{22}$ F. Brunet, R. Schaub, S. Fedrigo, R. Monot, J. Buttet, and W. Harbich, Surf. Sci. 512, 201 (2002).

${ }^{23}$ A. Tkatchenko, Phys. Rev. B 74, 035428 (2006).

${ }^{24}$ A. Tkatchenko, Phys. Rev. B 74, 235440 (2006).

${ }^{25}$ O. M. Magnussen, Chem. Rev. (Washington, D.C.) 102, 679 (2002).

${ }^{26}$ R. D. Diehl, T. Seyller, M. Caragiu, G. S. Leatherman, N. Ferralis, K. Pussi, P. Kaukasoina, and M. Lindroos, J. Phys.: Condens. Matter 16, 2839 (2004).

${ }^{27}$ W. A. Steele, Surf. Sci. 36, 317 (1973).

${ }^{28}$ A. Tkatchenko, N. Batina, and M. Galvan, Phys. Rev. Lett. 97, 036102 (2006). 
${ }^{29}$ D. J. Wales and J. P. K. Doye, J. Phys. Chem. A 101, 5111 (1997).

${ }^{30}$ D. M. Deaven and K. M. Ho, Phys. Rev. Lett. 75, 288 (1995).

${ }^{31}$ S. K. Gregurick, M. H. Alexander, and B. Hartke, J. Chem. Phys. 104, 2684 (1996).

${ }^{32}$ S. Kirkpatrick, C. D. Gilatt, and M. P. Vecchi, Science 220, 671 (1983).

${ }^{33}$ The stopping criteria for the conjugate-gradient (CG) optimizer is that the variation in energy after $10 \mathrm{CG}$ steps must be less than $10^{-5}$. Therefore, we say that two minima are different if the energy difference between them is less than $5 \times 10^{-5}$. Clearly, such approach can underestimate the number of minima; however, we have manually checked several "conflicting" configu- rations (with energy difference close to $5 \times 10^{-5}$ ) and found that the minima were close not only energetically but also geometrically.

${ }^{34}$ J. P. K. Doye and D. J. Wales, J. Chem. Phys. 116, 3777 (2002).

${ }^{35}$ D. J. Wales and J. P. K. Doye, J. Chem. Phys. 119, 12409 (2003).

${ }^{36}$ A. Tkatchenko and N. Batina, J. Chem. Phys. 122, 094705 (2005).

${ }^{37}$ K. Kern, R. David, R. L. Palmer, and G. Comsa, Phys. Rev. Lett. 56, 620 (1986).

${ }^{38}$ K. Kern, Phys. Rev. B 35, 8265 (1987).

${ }^{39}$ S. Torquato and F. H. Stillinger, J. Phys. Chem. B 105, 11849 (2001). 